\title{
Explaining the futility of the reproductive process in humans: past, present, and future
}

\author{
David F. Albertini ${ }^{1}$
}

Published online: 27 January 2017

(C) Springer Science+Business Media New York 2017

What proportions of conceptions succumb to the hazards of intrauterine development before a viable state is achieved?....and what are the hazards which cause this conceptional deficit? What moiety of the total have intrinsic, geneic or other, inevitable seeds of destruction in the fertilized gamete and what fraction succumb to an inhospitable or hostile intrauterine environment?

E.T. Engle, 1953

Taken from the preface of a treatise on the subject of pregnancy wastage, so was the matter of the "total postconceptional reproductive deficit" in humans articulated over six decades ago (Pregnancy Wastage, Ed., E. T. Engle 1953 Charles C. Thomas Publisher Springfield IL, USA). More telling perhaps were the roots of this publication proceedings of a conference sponsored by the Committee on Human Reproduction, National Research Council on behalf of the National Committee on Maternal Health, Inc. This meeting of the minds was held in New York City in the early 1950s and aimed to explain the loss of conceptions in humans based on the available data collected from luminaries of note. To traditionalists, hearing that Hertig, Rock, Corner, or Csapo were participants will come as no surprise. And complementing them were leading experts on animal reproduction who together first queried the apparent futility of human reproduction relative to the most thoroughly studied animal models of the day that included domesticated species, non-human primates and guinea pigs. This was the A-team of the time.

David F. Albertini

dalbertini@thechr.com

1 The Center for Human Reproduction, New York, NY, USA
As introduced last month, the vexing fact that from fertilization to birth, why the reproductive process in humans should be fraught with failure remains entrenched in the realms of the explainable and unexplainable. That the introduction of ARTs in today's overpopulated world has made even a modest contribution to our understanding of embryonic mortality and its management is arguable, as addressed this month in the report by Ghazal and Patrizio (Embryo wastage rates remain high in assisted reproductive technology (ART): a look at the trends from 2004 2013 in the USA. DOI 10.1007/s10815-016-0858). Clearly extending these kinds of analyses to other countries, and in the face of technologies being introduced to improve embryo selection, will provide an ever enlarging measure of just how well ARTs as practiced today and in the future cope with the inherent deficiencies in human reproduction recognized by our forebears.

But if history repeats itself, there is little reason for optimism. Consider that along the way to estimating the incidence of early pregnancy failure, which attended the introduction of the hCG assay (and antedated widespread adoption of ARTs), estimates of $70 \%$ of natural conceptions were believed to have no chance of developing to six weeks of gestation [1]. And with the greatly expanded knowledge base now at our disposal, how do our chances look in current terms? Again, not much seems to have changed. As Jarvis now reports, putting our best analytical tools and models for human fecundity to work reveals plausible estimates on the order of 40-60\% to explain the incidence of embryonic and fetal mortality over the spectrum from fertilization to birth [2]. A telling extension for these figures, reinforcing in even broader terms the nature and extent of the human reproductive condition, has recently been provided in the studies from Hardy and colleagues.

Perhaps the most significant associative factor aligning with pregnancy loss, as viewed through the lens of 
spontaneous abortion (SAB) is that of chromosomal abnormalities. While Engle and his colleagues could not have foreseen the magnitude of the "geneic" problem prior to the dawning of cytogenetics, recent accounting over the past 40 years representing five geographically diverse study sites makes clear that the incidence of SAB attributable to chromosomal abnormalities has remained pretty much unchanged [3]. Central to the significance of this work was the fact that culturing products of conception (POC) and the methods used to perform cytogenetic testing were maintained throughout the five series of materials analyzed covering a time period of some 40 years and including a total of 8319 SABs. Excepting for the apparent increased incidence of trisomies over time, revealed to be due to increased maternal age in the later study groups, this body of work reaffirmed in the author's own words "that the primary determinants of chromosome abnormality are hard-wired into our species."

Interestingly, the patterns of specific chromosomes implicated in SABs are not unlike that observed in trophectoderm biopsies of ART produced blastocysts [4] and are comparable to what is found in SABs of an infertile population of women [5]. Collectively, then, it seems that our genetics are at play in the everyday world of pregnancy failure, independent of whether conceptions are a byproduct of natural mating, or ART mediation for the subfertile-waiting-to-be-infertile seeking medical intervention.

There is much more for our readership to glean from this month's issue, featuring on the cover a glimpse of Arthur Hertig's human ovum sketch dating back to 1967. For example, the review by Hanson and colleagues provides a provocative and prescient perspective of the larger domain within which our myopic management of female infertility exists (Female infertility, infertility-associated diagnoses, and comorbidities: a review 10.1007/s10815-016-0836). Extending the conversation, and consequences, of our obsession with cryopreservation are several contributions aimed at broadstroked acceptance versus selective utilization (Freeze-all cycle for all normal responders? 10.1007/s10815-016-0834), documentation of long term effects on offspring (Increased risk of large-for-gestational age birthweight in singleton siblings conceived with in vitro fertilization in frozen versus fresh cycles 10.1007/s10815-016-0850) and an accompanying commentary by Rinaudo and $\mathrm{Hsu}$ encouraging deliberation in this fast moving arena of ARTs (To freeze or not to freeze: heating the debate but cooling the practice? DOI 10.1007/s10815-017-0870).

Finally, our coverage this month takes on the question of where the guiding principles for future ARTs come from that will shape best practices in the near and distant future. It is time to take a fresh look at a research enterprise plagued by inconsistencies in study design, limitations due to patient variance, and an alltoo-obvious tendency to overlook the importance of rigorous standards and uniformity of measurement necessitated in the tailoring and interpretation of good science! Accordingly, we welcome two reports illustrating a change in mindset whose adoption is a welcomed one as research in human ARTs move forward.

First, the paper by Weiss and colleagues refines and defines what a biobank would be when put into practice (Biobanking of different body fluids within the frame of IVF — a standard operating procedure to improve reproductive biology research. 10.1007/s10815-016-0847). Next, in view of the increasing role of ovarian tissue cryopreservation in fertility preservation, comes the contribution from The Oncofertility Consortium setting the bar for standardization of practices that would be well-advised generally in human ARTs research (Good manufacturing practice requirements for the production of tissue vitrification and warming and recovery kits for clinical research. 10.1007/s10815-016-0846).

In the end, defining reproductive fitness, and all of its nuances, remains an elusive goal notwithstanding our focus on the "geneic." Taking heed of the "moiety of the total" in Engle's words will as all signs show, extend well beyond the realm of Mendelian genetics, as the purveyors of epigenetics continue to have their say [6].

\section{References}

1. Boklage CE. Survival probability of human conceptions from fertilization to term. Int J Fertil. 1990;35(2):75, 9-80, 1-94.

2. Jarvis GE. Estimating limits for natural human embryo mortality. F1000Res. 2016;5:2083.

3. Hardy K, Hardy PJ, Jacobs PA, Lewallen K, Hassold TJ. Temporal changes in chromosome abnormalities in human spontaneous abortions: results of 40 years of analysis. Am J Med Genet A. 2016;170(10):2671-80.

4. Franasiak JM, Forman EJ, Hong KH, Werner MD, Upham KM, Treff NR, et al. Aneuploidy across individual chromosomes at the embryonic level in trophectoderm biopsies: changes with patient age and chromosome structure. J Assist Reprod Genet. 2014;31(11): 1501-9.

5. Werner M, Reh A, Grifo J, Perle MA. Characteristics of chromosomal abnormalities diagnosed after spontaneous abortions in an infertile population. J Assist Reprod Genet. 2012;29(8):817-20.

6. Haig D. Maternal-fetal conflict, genomic imprinting and mammalian vulnerabilities to cancer. Philos Trans R Soc Lond Ser B Biol Sci. 2015;370(1673). 